\title{
Methotrexate vs Azathioprine in Second-line Therapy of Sarcoidosis
}

\author{
Adriane D. M. Vorselaars, MD; Wim A. Wuyts, MD, PhD; Veronique M. M. Vorselaars, MD; \\ Pieter Zanen, MD, PhD; Vera H. M. Deneer, PhD; Marcel Veltkamp, MD, PhD; \\ Michiel Thomeer, MD, PhD; Coline H. M. van Moorsel, PhD; \\ and Jan C. Grutters, MD, PhD
}

\begin{abstract}
Background: Steroids remain the first-choice therapeutic in sarcoidosis; however, long-term use is associated with toxicity. Evidence defining the best second-line therapeutic is currently lacking. The aim of this study was to compare the effect of methotrexate and azathioprine on prednisone tapering, pulmonary function, and side effects in the second-line treatment of sarcoidosis.

Methods: An international retrospective cohort study was performed, reviewing all patients with sarcoidosis who started methotrexate or azathioprine until 2 years after initiation or discontinuation. A linear mixed model with $\mathrm{FEV}_{1}$, vital capacity (VC), diffusing capacity of lung for carbon monoxide (DLCO), and prednisone dose changes over time as end points was used. Side effects were compared with $\chi^{2}$ tests.

Results: Two hundred patients were included, of whom 145 received methotrexate and 55 azathioprine. Prednisone daily dose decreased a mean of $6.32 \mathrm{mg} / \mathrm{y}(P<.0001)$ while on therapy, with a similar steroid-sparing capacity for methotrexate and azathioprine. Of all patients completing 1 year of therapy, $70 \% \mathrm{had}$ a reduction in daily prednisone dose of at least $10 \mathrm{mg}$. FEV 1 showed a mean increase of $52 \mathrm{~mL} / \mathrm{y}(P=.006)$ and VC of $95 \mathrm{~mL} / \mathrm{y}(P=.001)$ in both treatment groups. DLCo \% predicted increased, with a mean of $1.23 \% / y(P=.018)$. There were more patients with infections in the azathioprine group $(34.6 \%$ vs $18.1 \%, P=.01)$, but no differences regarding other side effects.

Conclusions: This retrospective study comparing the effect of second-line therapy in sarcoidosis shows that both methotrexate and azathioprine have significant steroid-sparing potency, a similar positive effect on lung function, and comparable side effects, except for a higher infection rate in the azathioprine group.

CHEST 2013; 144(3):805-812
\end{abstract}

Abbreviations: $\mathrm{DLCO}=$ diffusing capacity of lung for carbon monoxide; $\mathrm{RCT}=$ randomized controlled trial; $\mathrm{VC}=\mathrm{vital}$ capacity

$\mathbf{S}$ arcoidosis is a systemic, granulomatous disease that can affect multiple organs and has a variable clinical course. Although new therapies are slowly emerging, steroids remain the mainstay of therapy. ${ }^{1,2}$ Long-term steroid use, however, is associated with severe toxicity (eg, weight gain, diabetes, osteoporosis). In cases of intolerable side effects, inefficacy, or prolonged use of steroids, a second-line therapeutic is added to the treatment regimen. However, evidence defining the best second-line therapeutic currently is unavailable because studies comparing different treatment options are lacking.

Methotrexate often is considered the first choice in second-line therapeutics, ${ }^{3-5}$ but evidence is limited. Methotrexate was reported to be effective in small case series, ${ }^{6-8}$ and a single, small, randomized controlled trial ( RCT) in 24 patients showed a steroid-sparing effect in acute sarcoidosis. ${ }^{9}$ A retrospective cohort study in 105 patients found a significant steroid-sparing effect and improvement in lung function or other affected organs in $66 \%$ of 50 patients with sarcoidosis who completed at least 2 years of methotrexate therapy. ${ }^{10}$

Azathioprine is another second-line therapeutic for refractory sarcoidosis. ${ }^{3,4}$ To date, no RCTs or large case series are available. Azathioprine has been reported to be effective in small case series and case reports, with a maximum of 11 patients. ${ }^{11-13}$

New, promising, but expensive treatment options, such as biologics, are arising. ${ }^{14}$ However, in current 
times of rising health-care costs, the existing and lessexpensive treatment options for sarcoidosis should be fully explored. The efficacy of methotrexate vs azathioprine as first-choice second-line therapy in sarcoidosis has not been studied thus far. Therefore, we studied the effect of methotrexate and azathioprine regarding prednisone tapering, pulmonary function tests, and side effects.

\section{Materials AND Methods}

\section{Study Design}

This international retrospective cohort study compared two different second-line therapeutics for refractory sarcoidosis. Patients were recruited from the University Hospitals Leuven, Belgium, and St Antonius Hospital, Nieuwegein, The Netherlands. Both hospitals are tertiary referral centers for interstitial lung disease in their respective countries. In Belgium, the first drug of choice in second-line therapy of sarcoidosis historically has been azathioprine, whereas in The Netherlands, it is methotrexate.

Medical records were reviewed for demographic data, organ involvement, and radiographic scadding stage. Patients were divided into pulmonary and extrapulmonary treatment subgroups according to the reason for initiation of second-line therapy. Patients in the extrapulmonary treatment group could also have mild pulmonary localizations of sarcoidosis. Prednisone dosage and side effects were collected together with the pulmonary function parameters of total lung capacity, $\mathrm{FEV}_{1}$, vital capacity $(\mathrm{VC})$, and diffusing capacity of lung for carbon monoxide (DLCO). Pulmonary function parameters were included from 1 year before the start of therapy until 2 years after treatment initiation. Follow-up of patients ended at discontinuation of second-line therapy, at the start of third-line therapy, or after a maximum of 2 years on second-line therapy. Clinical routine and follow-up protocols were comparable in both centers. We obtained a declaration of no objection from the local institutional review board with registration number LTME/Z-12.05 and acronym METHVERAZ.

\section{Patients}

All patients who started methotrexate at St Antonius Hospital between June 2004 and September 2011 were selected. Patients

Manuscript received July 11, 2012; revision accepted March 7, 2013.

Affiliations: From the Center of Interstitial Lung Diseases (Drs A. D. M. Vorselaars, V. M. M. Vorselaars, Veltkamp, van Moorsel, and Grutters), Department of Pulmonology, and Department of Clinical Pharmacy (Dr Deneer), St Antonius Hospital, Nieuwegein, The Netherlands; Unit for Interstitial Lung Diseases (Dr Wuyts), Department of Pulmonology, University Hospitals Leuven, Leuven, Belgium; Division of Heart and Lungs (Drs Zanen, van Moorsel, and Grutters), University Medical Centre Utrecht, Utrecht, The Netherlands; and the Department of Respiratory Medicine (Dr Thomeer), Hospital Oost Limburg, Research Cluster Oncology, UHasselt, Hasselt, Belgium.

Funding/Support: The authors have reported to CHEST that no funding was received for this study.

Correspondence to: Jan C. Grutters, MD, PhD, Center of Interstitial Lung Diseases, Department of Pulmonology, St Antonius Hospital, Koekoekslaan 1, 3435 CM Nieuwegein, The Netherlands; e-mail: j.grutters@antoniusziekenhuis.nl

(c) 2013 American College of Chest Physicians. Reproduction of this article is prohibited without written permission from the American College of Chest Physicians. See online for more details. DOI: $10.1378 /$ chest.12-1728 who started azathioprine between November 1995 and July 2011 at the University Hospitals Leuven were selected. Sarcoidosis was diagnosed according to the American Thoracic Society, European Respiratory Society, and World Association for Sarcoidosis and Other Granulomatous Disorders statement on sarcoidosis. ${ }^{15}$

Patients eligible for second-line therapy had persistent active sarcoidosis with impaired organ function or severely diminished quality of life. Furthermore, they were unresponsive to steroids, experienced severe side effects, or had other serious contraindications for steroid use. All patients were naive to second-line therapy.

Patients received methotrexate according to a standard protocol that started at $10 \mathrm{mg} / \mathrm{wk}$ and increased to $15 \mathrm{mg} / \mathrm{wk}$ with regular checks of hepatic function. Additionally, all patients used $5 \mathrm{mg}$ folic acid. Azathioprine $2 \mathrm{mg} / \mathrm{kg}$ body weight was administered, with a maximum of $150 \mathrm{mg} / \mathrm{d}$ and routine checks of hepatic function and blood counts. Tapering of prednisone was the physician's decision and not part of an established tapering schedule.

\section{Statistical Analysis}

Student $t$ test was used to compare means of normally distributed variables, and $\chi^{2}$ test was used for categorical variables. Treatment effects on pulmonary function and prednisone dosage were calculated with a linear mixed model, with $\mathrm{FEV}_{1}, \mathrm{VC}$, DLCO, and prednisone dose over time as primary end points..$^{16}$ European Respiratory Society standard prediction equations for pulmonary function were used in both hospitals. Covariates and factors (eg, ethnicity, treatment indication) were inserted into the model to detect confounders. The -2 restricted $\log$ likelihood values were used to evaluate whether insertion improved the fit of the model significantly. Pulmonary function tests were corrected for height, sex, and age in the linear mixed model. The differences in pulmonary function values between the pretreatment period and during treatment were assessed with the linear mixed model by inserting a pretreatment $(0)$ and posttreatment (1) initiation variable. We also inserted a treatment $X$ time interaction to assess whether the lung function continued to increase over time (see e-Appendix 1 for a detailed description). Differences in side effects were estimated with $\chi^{2}$ tests.

To determine the number of patients responsive to therapy, a subanalysis was performed, including all patients completing 1 year of therapy. Change in pulmonary function parameters and daily prednisone intake were calculated with $\chi^{2}$ tests.

Statistical analysis was performed with SPSS for Windows version 17.0 (IBM), and graphs were created with GraphPad Prism 5.0 (GraphPad Software, Inc) software. $P<.05$ was considered significant.

\section{RESULTS}

\section{Patients}

A total of 200 patients were included in this study; 145 patients started methotrexate therapy, and 55 started azathioprine therapy. Baseline characteristics of both groups are shown in Table 1. There were significantly more nonwhite patients in the methotrexate cohort $(P=.004)$, and the methotrexate group had a higher DLCO at baseline $(P=.01)$. Extrapulmonary treatment indications were cardiac sarcoidosis, neurosarcoidosis, joint sarcoidosis, uveitis, and disabling fatigue. The group with a multiple organ indication comprised patients with various symptoms of extrapulmonary origin. 
Table 1-Baseline Characteristics of Treatment Cohort

\begin{tabular}{|c|c|c|c|c|}
\hline Characteristic & All Patients ${ }^{\mathrm{a}}(\mathrm{N}=200)$ & Methotrexate $(\mathrm{n}=145)$ & Azathioprine $(\mathrm{n}=55)$ & $P$ Value \\
\hline Age at start of therapy, $y$ & $47.4 \pm 10.6$ & $47.4 \pm 10.1$ & $47.2 \pm 12.1$ & NS \\
\hline Male sex & $126(63)$ & $90(62.1)$ & $36(65.5)$ & NS \\
\hline Race & & & & 0.004 \\
\hline White & $180(90)$ & $125(86.2)$ & $55(100)$ & \\
\hline Black & $13(6.5)$ & $13(9.0)$ & $0(0)$ & \\
\hline Other & $7(3.5)$ & $7(4.8)$ & $0(0)$ & \\
\hline $\mathrm{BMI}, \mathrm{kg} / \mathrm{m}^{2}$ & $27.4 \pm 4.4$ & $27.5 \pm 4.4$ & $27.1 \pm 4.4$ & NS \\
\hline Smoking & & & & NS \\
\hline Current & $15(8)$ & $10(7)$ & $5(10)$ & \\
\hline Former & $72(36)$ & $51(39)$ & $21(42)$ & \\
\hline Histology & $181(91)$ & $133(95)$ & $48(92)$ & NS \\
\hline Disease duration, mo & $59.7 \pm 87.2$ & $61.1 \pm 85.1$ & $56.3 \pm 93.4$ & NS \\
\hline Scadding stage & & & & NS \\
\hline 0 & $18(9)$ & $16(11)$ & $2(4)$ & \\
\hline $\mathrm{I}$ & $22(11)$ & $13(9)$ & $9(17)$ & \\
\hline II & $83(41.5)$ & $58(40)$ & $25(47)$ & \\
\hline III & $45(22.5)$ & $31(22)$ & $14(26)$ & \\
\hline IV & $29(14.5)$ & $26(18)$ & $3(6)$ & \\
\hline Previous therapy & & & & NS \\
\hline Prednisone & $173(87)$ & $121(85)$ & $52(96)$ & \\
\hline Prednisone + hydroxychloroquine & $11(6)$ & $11(8)$ & $0(0)$ & \\
\hline None (contraindication prednisone) & $13(7)$ & $11(8)$ & $2(4)$ & \\
\hline Prednisone dose in patients using at start & $20.5 \pm 13.2$ & $20.0 \pm 12.6$ & $21.6 \pm 10.2$ & NS \\
\hline \multicolumn{5}{|l|}{ PFT, $\%$ predicted } \\
\hline TLC & $79.1 \pm 17.3$ & $78.5 \pm 16.7$ & $80.6 \pm 18.9$ & NS \\
\hline VC & $85.1 \pm 20.6$ & $86.9 \pm 20.3$ & $80.3 \pm 20.7$ & NS \\
\hline $\mathrm{FEV}_{1}$ & $75.1 \pm 22.4$ & $76.1 \pm 22.8$ & $72.3 \pm 21.1$ & NS \\
\hline DLCO & $64.7 \pm 16.8$ & $66.6 \pm 17.2$ & $59.7 \pm 14.7$ & .01 \\
\hline Treatment indication & & & & NS \\
\hline Pulmonary & $136(68)$ & $95(66)$ & $41(74)$ & \\
\hline Neurologic & $15(7.5)$ & $10(6.9)$ & $5(9.1)$ & \\
\hline Cardiac & $9(4.5)$ & $6(4.1)$ & $3(5.5)$ & \\
\hline Uveitis & $2(1)$ & $2(1.4)$ & $0(0)$ & \\
\hline Joints & $10(5)$ & $7(4.8)$ & $3(5.5)$ & \\
\hline Fatigue & $5(2.5)$ & $4(2.8)$ & $1(1.8)$ & \\
\hline Cutaneous & $2(1)$ & $1(0.7)$ & $1(1.8)$ & \\
\hline GI & $1(0.5)$ & $1(0.7)$ & $0(0)$ & \\
\hline Renal & $1(0.5)$ & $1(0.7)$ & $0(0)$ & \\
\hline Multiple organs & $19(9.5)$ & $18(12.4)$ & $1(1.8)$ & \\
\hline Tertiary referral & $150(75)$ & $113(79)$ & $37(69)$ & NS \\
\hline
\end{tabular}

Data are presented as mean \pm SD or No. $(\%)$. A subdivision is made between patients treated with methotrexate and azathioprine. $P$ values are given for significant differences between the two groups. DLCO = diffusing capacity of lung for carbon monoxide; NS = not significant; $\mathrm{PFT}=$ pulmonary function test TLC $=$ total lung capacity; $\mathrm{VC}=$ vital capacity.

anly eight of 145 patients in the methotrexate group were treated in Belgium, and only three of 55 patients with the azathioprine group were treated in The Netherlands.

Figure 1 shows the 2-year follow-up of all patients receiving methotrexate and azathioprine. Of 37 patients in whom second-line therapy was stopped because of side effects, 19 were not stable and started another second-line therapeutic or third-line therapy. Twelve patients remained stable with the use of steroids, which were in some cases increased in dose again. Six patients were lost to follow-up.

\section{Prednisone Use}

Prednisone daily dosing regimen decreased a mean of $6.32 \mathrm{mg} / \mathrm{y}(\mathrm{SE}, 0.63 ; P<.0001)$ while on therapy, with a similar effect on prednisone regimen in both treatment arms (Fig 2). Prednisone tapering in the pulmonary and extrapulmonary subgroups was also similar.

A subanalysis of all patients completing 1 year of follow-up who used prednisone at baseline $(\mathrm{n}=100)$ showed a decrease in prednisone intake of $>10 \mathrm{mg} / \mathrm{d}$ in $70(70 \%)$, with no significant differences between methotrexate and azathioprine. Characteristics of this group are shown in Table 2.

\section{Pulmonary Function Tests}

We noted significantly higher pulmonary function test results after the start of treatment compared with 


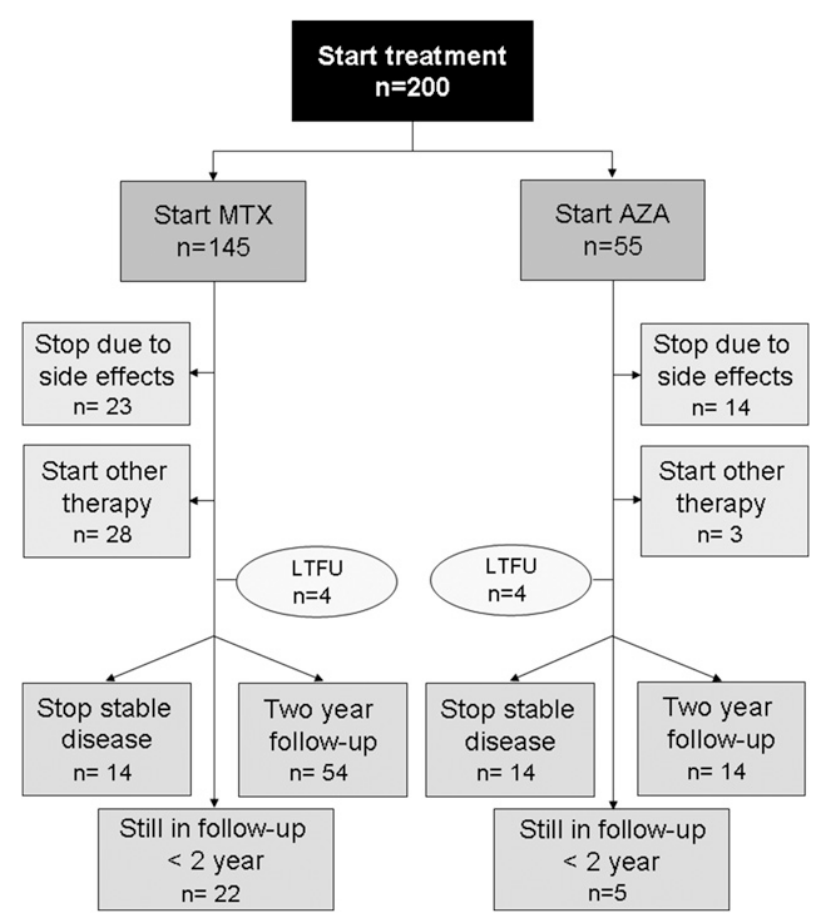

FIGURE 1. Flowchart of patients who received MTX and AZA during a 2-y follow-up from start of treatment. All patients receiving treatment were included in the analysis. $\mathrm{AZA}=$ azathioprine; $\mathrm{LTFU}=$ loss to follow-up; MTX $=$ methotrexate.

the year before treatment initiation. This difference was $81 \mathrm{~mL}$ (2.94\% predicted) in $\mathrm{FEV}_{1}(P=.001), 97 \mathrm{~mL}$ (2.57\% predicted) in $\mathrm{VC}(P=.004)$, and $0.25 \mathrm{mmol} / \mathrm{kPa} / \mathrm{s}$ ( $1.5 \%$ predicted $)$ in DLCO $(P=.002)$.

After initiation of treatment, $\mathrm{FEV}_{1}$ showed a yearly increase during treatment of $52 \mathrm{~mL}(2.17 \%$ predicted, $P=.006)$, whereas VC showed a yearly increase of $95 \mathrm{~mL}$ (2.81\% predicted, $P=.001)$. The mean increase in $\mathrm{FEV}_{1}$ and $\mathrm{VC}$ was similar in both the azathioprine and the methotrexate groups (Figs 3A, 3B). DLCO showed a yearly increase of $0.107 \mathrm{mmol} / \mathrm{kPa} / \mathrm{s}(1.23 \%$ predicted, $P=.018)$ after treatment initiation. Mean

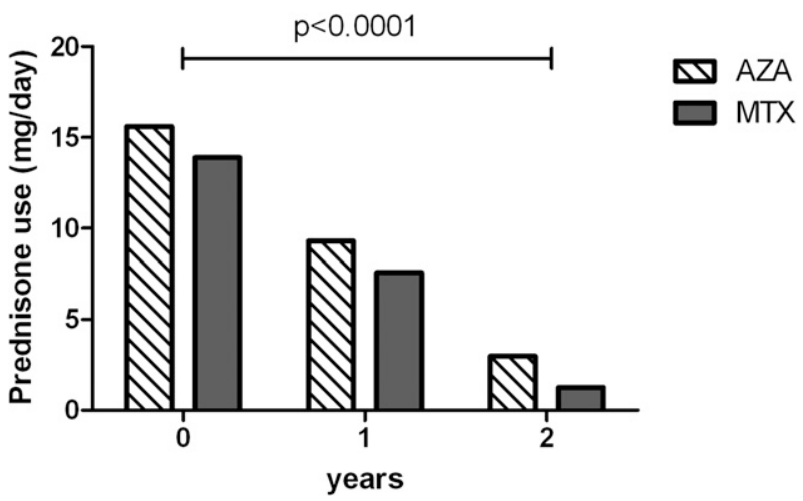

Figure 2. Effect of AZA and MTX on daily prednisone dose. Daily prednisone use decreased with $6.32 \mathrm{mg} /$ year (SE, 0.63; $P<.0001)$. The difference between AZA and MTX was not significant $(P=.139)$. See Figure 1 legend for expansion of abbreviations.
DLCO was $0.61 \mathrm{mmol} / \mathrm{kPa} / \mathrm{s}$ (5.12\% predicted) lower in the azathioprine group with borderline significance $(P=.049)$, but this difference was stable over time (Fig 3C).

The group with nonpulmonary treatment indication had an overall $770-\mathrm{mL}$ (23.5\% predicted) higher $\mathrm{FEV}_{1}$, an overall $491-\mathrm{mL}$ ( $13.1 \%$ predicted) higher VC, and a $13.3 \%$ higher DLCO than the pulmonary treatment group $(P<.0001)$. There was no difference in treatment effect between the group with the pulmonary and the group with the nonpulmonary treatment indication. Ethnicity and DLCO at baseline were not confounding in the analysis of any of the pulmonary function parameters.

A subanalysis of all patients completing 1 year of follow-up while receiving treatment in whom pulmonary function tests were available $(\mathrm{n}=100)$ showed that $57(57 \%)$ had an improvement of $\geq 10 \%$ in at least one of the parameters $\left(\mathrm{FEV}_{1}, \mathrm{VC}\right.$, and DLCO), as shown in Table 2. For each parameter separately, these numbers were $36 \%$ of patients for $\mathrm{FEV}_{1}, 38 \%$ for VC, and 37\% for DLCO. There were no significant differences between azathioprine and methotrexate for any of these response outcomes.

\section{Side Effects}

The most frequently reported side effects were infections in 44 patients (22.4\%) (Fig 4). All infections requiring antibiotics, hospital admission, or (temporary) discontinuation of treatment were included. There was a significant difference in the number of patients having infections in the azathioprine group vs the methotrexate group $(34.6 \%$ vs $18.1 \%, P=.01)$. Respiratory infections requiring antibiotics comprised the majority (30 patients); four patients experienced varicella zoster virus (all in methotrexate group). Furthermore, one case of empyema and two cases of sepsis (all in azathioprine group) occurred. GI problems were reported by 37 patients $(19.0 \%)$ patients and were the most common reason for patients to quit treatment because of nausea, stomachache, and diarrhea. Severe hepatic function decline requiring alteration or discontinuation of treatment was found in 14 patients $(7.2 \%)$; however, liver function recovered in all patients after discontinuation of treatment. Other repeatedly reported side effects were headache $(4.1 \%)$ and malaise $(7.7 \%)$. There were no differences in the occurrence of these side effects between the two treatment groups. Thirtyseven patients $(18.5 \%)$ had to discontinue therapy because of side effects; this occurred most often within the first 3 months. The dropout rates because of side effects were not significantly different between treatment groups. Rarely seen side effects (two or fewer patients) were hair loss, impotence, gingivitis, decline in renal function, anemia requiring transfusion, and 
Table 2-Subanalysis of 123 Patients Completing 1 y of Follow-up on Second-line Therapy

\begin{tabular}{|c|c|c|c|c|}
\hline All Patients & Total Group & Methotrexate & Azathioprine & $P$ Value \\
\hline 10 -mg reduction of prednisone after $1 \mathrm{y},{ }^{\mathrm{a}} \%$ & 70 & 65 & 80 & NS \\
\hline Increase $\mathrm{FEV}_{1}, \%$ predicted & $8.3 \pm 14.4$ & $7.6 \pm 14.4$ & $10.0 \pm 14.5$ & NS \\
\hline Increase VC, \% predicted & $8.8 \pm 13.5$ & $9.2 \pm 13.1$ & $7.8 \pm 14.7$ & NS \\
\hline Increase DLCO, $\%$ predicted & $8.7 \pm 17.8$ & $6.6 \pm 11.0$ & $13.4 \pm 27.2$ & NS \\
\hline Increase $>10 \%$ any PFT, $\%$ & 57.0 & 56.3 & 58.6 & NS \\
\hline \multicolumn{5}{|l|}{ Patients with pulmonary treatment indication } \\
\hline Increase VC, \% predicted & $10.6 \pm 14.7$ & $10.5 \pm 14.7$ & $10.9 \pm 15.6$ & NS \\
\hline$>10 \%$ VC increase, $\%$ & 45.5 & 42.2 & 52.4 & NS \\
\hline
\end{tabular}

Data are presented as mean \pm SD unless otherwise indicated. See Table 1 legend for expansion of abbreviations.

${ }^{a}$ Of patients using prednisone at baseline, $\mathrm{n}=100$.

thrombocytopenia. In the cohort, only two patients had leukopenia requiring dose adjustment or discontinuation of the second-line drug; both were in the azathioprine group. Two patients in the methotrexate

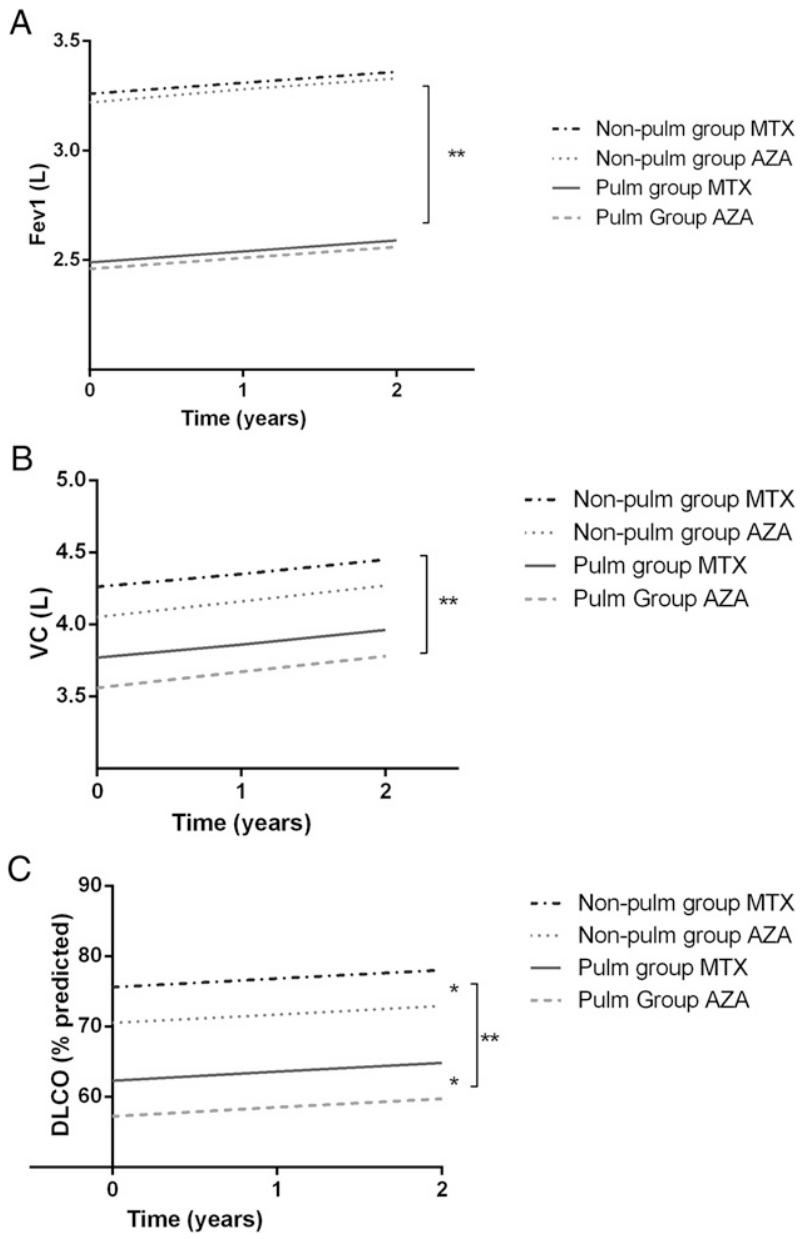

FiguRE 3. A-C, Linear mixed-model pulmonary function parameters over time. Pulmonary function increased significantly over time for $\mathrm{FEV}_{1}(P=.006)(\mathrm{A})$, VC $(P=.001)(\mathrm{B})$, and DLCO $(P=.018)(\mathrm{C})$. There was a significant difference between the pulm and non-pulm treatment groups. No difference was found between the AZA and MTX treatment effect. ${ }^{*} P=.049$. ${ }^{*} P<<.001$. DLCO = diffusing capacity of lung for carbon monoxide; Nonpulm = nonpulmonary; Pulm = pulmonary; $\mathrm{VC}=$ vital capacity . See Figure 1 legend for expansion of other abbreviations. group were hospitalized for pneumonia compared with four patients in the azathioprine group. One of these patients was treated for Pneumocystis jiroveci pneumonia. There were no patients with a fatal outcome while receiving second-line therapy during the 2 years of follow-up.

\section{Discussion}

This study shows that both methotrexate and azathioprine have significant steroid-sparing potency, a similar positive effect on lung function, and comparable side effects, except for a higher infection rate in the azathioprine group. We found a decrease of prednisone daily dosing regimen of $6.32 \mathrm{mg} / \mathrm{y}$ while receiving therapy, with no differences between drugs. Seventy percent of all patients completing 1 year of follow-up had a decrease of at least a 10-mg daily dose. The results regarding methotrexate confirm those of Baughman et al, ${ }^{9}$ who found a significant reduction of prednisone use after 7 months in an RCT comparing methotrexate with placebo in 24 patients. Lower and Baughman ${ }^{10}$ found a reduction of $>10 \mathrm{mg} / \mathrm{d}$ prednisone in $80 \%$ of 30 patients treated with methotrexate who were followed retrospectively for 2 years, which is in line with the present findings. Only three case series ${ }^{11-13}$ with a maximum of 11 patients with sarcoidosis

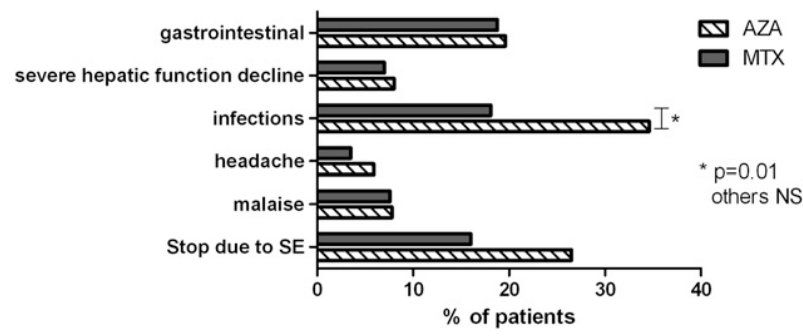

FIgURE 4. Reported side effects during AZA and MTX treatment by percentage of patients. Patients could have more than one side effect. Hepatic function decline was defined severe when it was a reason to discontinue therapy. There was a significant difference between the number of patients with infections in both treatment groups $(P=.01)$. NS = not significant. See Figure 1 legend for expansion of other abbreviations. 
described the effect of azathioprine on lung function or prednisone dose without quantification of effect, making comparison with the present study difficult.

We found a mean increase in of $52 \mathrm{~mL} / \mathrm{y}$ in $\mathrm{FEV}_{1}$ and $95 \mathrm{~mL} / \mathrm{y}$ in $\mathrm{VC}$, with no difference between the two drugs for either. DLco \% predicted increased, with a mean of $1.23 \% / y$. The only RCT for methotrexate in sarcoidosis did not show an improvement in pulmonary function, which could have been a result of a power problem because the study was conducted in only 24 patients. ${ }^{9}$ Lower and Baughman ${ }^{10}$ showed that 22 of 50 patients using methotrexate for 2 years had an improvement in $\mathrm{VC}$ of $>10 \%$, which is in line with $38 \%$ in the present cohort. Vucinic ${ }^{8}$ showed an improvement of unspecified magnitude in $\mathrm{FEV}_{1}$ in $80 \%$ and DLCO in $65 \%$ of 91 patients with sarcoidosis while taking methotrexate for 6 months. After 1 year of treatment, we found improvement in $\mathrm{FEV}_{1}$ in $74 \%$ of patients and DLCO in $68 \%$ of patients. Because the size of the improvement was not quantified by Vucinic, the clinical relevance of this improvement remains uncertain so far. Hence, this study is the first to our knowledge to describe the treatment effect of both azathioprine and methotrexate on pulmonary function in a large cohort of patients.

With available data from before initiation of methotrexate or azathioprine, we were able to detect a small but significant increase in pulmonary function. A yearly drop in lung function would be expected because of natural evolution even in healthy control subjects, let alone in patients with severe sarcoidosis. Therefore, we conclude that therapy caused improvement or at least stabilization of pulmonary function. Unexpected was the equal increase in pulmonary function in both the extrapulmonary and the pulmonary treatment groups. Although pulmonary function was not the main indication for initiating treatment in this group, most patients still had pulmonary localization (eg, hilar lymphadenopathy) with or without symptoms.

A total of 37 patients (18.5\%) had to discontinue therapy because of side effects. This dropout rate is higher than that described by Lower and Baughman, ${ }^{10}$ where $9.5 \%$ of 105 patients quit methotrexate because of side effects or noncompliance. Vucinic ${ }^{8}$ found that 0 of 91 patients had to discontinue methotrexate because of side effects in 6 months of treatment. These lower dropout rates related to side effects might be explained by the lower dosing regimen used in these studies (10 vs $15 \mathrm{mg} / \mathrm{wk}$ ). Studies comparing side effects of azathioprine and methotrexate in other diseases include a case-control study in $>23,000$ patients with rheumatoid arthritis, showing a moderate increased risk of severe infections in those taking azathioprine. ${ }^{17}$ However, a 3-year case-control study of 53 patients with rheumatoid arthritis revealed more-frequent adverse effects in the methotrexate group but a sim- ilar withdrawal rate in both groups ${ }^{18}$ and an RCT in 126 patients with antineutrophil cytoplasmic antibody-associated vasculitis showed no differences in occurrence of adverse events between drugs. ${ }^{19}$

To date, this study is the first to our knowledge to compare the steroid-sparing effect, the effect on pulmonary function, and side effects of azathioprine and methotrexate therapy in patients with sarcoidosis. In addition, this cohort is the largest of second-line therapy in this patient population described thus far. Baughman and Lower ${ }^{20}$ found that two-thirds of patients in their review responded to methotrexate, and onehalf responded to azathioprine. However, the majority of patients receiving azathioprine had previously been treated with methotrexate, making a comparison that defined the best second-line therapeutic impossible. On the other hand, their findings suggest that patients not responsive to one second-line therapeutic might still benefit from changing to another second-line therapeutic.

Present day studies in sarcoidosis therapeutics often are initiated by pharmaceutical companies that are investigating new drugs, whereas the existing and lessexpensive options have not been fully explored. In The Netherlands and Belgium, a treatment regimen of azathioprine $100 \mathrm{mg} / \mathrm{d}$ costs $€ 84$ to $€ 146$ per patient per year; treatment with methotrexate $15 \mathrm{mg} / \mathrm{wk}$ costs $€ 58$ to $€ 83$ per patient per year. ${ }^{21,22}$ These costs are insignificant when compared with the high costs of treatment with biologics (eg, infliximab, adalimumab), which start at $€ 20,000$ per patient for 6 months.

One of the limitations of this study is the retrospective design because nonstandardization of follow-up necessarily occurs in a retrospective study. As a result, we might have missed events or side effects; nevertheless, through systematic and thorough investigation of patient records, we have tried to minimize the loss of follow-up and data. The absence of a placebo group makes it impossible to fully attribute the observed steroid-sparing effect to the therapeutic intervention as opposed to resolution of sarcoidosis regardless of drug therapy. However, we believe that this is a reflection of daily practice in clinical care because both hospitals in The Netherlands and Belgium had the same mean prednisone dose at the start of second-line therapy (20 $\mathrm{mg}$ for patients receiving prednisone, $15 \mathrm{mg}$ for the total cohort). Furthermore, the disease duration before initiating second-line therapy was the same in both groups (mean, 5 years), indicating that this cohort comprises patients with chronic and often severe sarcoidosis in whom second-line therapy was not a first refuge. Therefore, we believe that it would be too simplistic to attribute the improvement in patients of both centers to the natural history of sarcoidosis.

This large international retrospective cohort study adds important information to the medical literature, 
especially regarding azathioprine and its role in sarcoidosis compared with methotrexate. The major strength of this study is that each drug was a first-choice second-line therapeutic at a different institution. Therefore, there was no inherent severity selection or symptomatic bias toward using one or the other drug. The study design might have caused bias in the number of patients in whom third-line agents were initiated (Fig 1) because patients in The Netherlands were more likely to receive infliximab, a drug that is not available in Belgium.

We found significantly more patients with infections requiring antibiotics or drug tapering in the azathioprine group $(34.6 \%$ vs $18.1 \%, P=.01)$, with no significant differences regarding other side effects. The observed difference might be a reflection of different antibiotic regimens in both countries, with The Netherlands being cautious with use of antibiotics compared with the global standard..$^{23}$ Another option might be that $P$ jiroveci pneumonia prophylaxis was only introduced halfway into the inclusion period in the azathioprine group, which currently is routine practice in this group. We did not perform thiopurine methyltransferase genotyping 24 in the patients treated with azathioprine.

In conclusion, this study is the first to compare the effect of methotrexate and azathioprine in second-line sarcoidosis treatment. Although less is known about azathioprine than methotrexate in sarcoidosis, the study shows that both drugs are equally effective in terms of pulmonary function improvement and have a significant steroid-sparing effect. Therefore, both methotrexate and azathioprine are suitable options as second-line therapy of chronic sarcoidosis.

\section{ACKNOWLEDGMENTS}

Author contributions: Drs A. D. M. Vorselaars and Grutters had full access to all the data in the study and take responsibility for the integrity of the data and the accuracy of the data analysis, including and especially any adverse effects.

Dr A. D. M. Vorselaars: contributed to the study concept and design, patient recruitment and data collection, data analysis and interpretation, manuscript preparation, and manuscript drafting and final approval.

Dr Wuyts: contributed to the study concept and design, patient recruitment and data collection, data analysis and interpretation, and manuscript drafting and final approval.

Dr V. M. M. Vorselaars: contributed to patient recruitment and data collection, data analysis and interpretation, manuscript preparation, and manuscript drafting and final approval.

Dr Zanen: contributed to data analysis and interpretation, manuscript preparation, and manuscript drafting and final approval. Dr Deneer: contributed to data analysis and interpretation and manuscript drafting and final approval.

Dr Veltkamp: contributed to patient recruitment and data collection, data analysis and interpretation, and manuscript drafting and final approval.

Dr Thomeer: contributed to patient recruitment and data collection, data analysis and interpretation, and manuscript drafting and final approval.
Dr van Moorsel: contributed to data analysis and interpretation and manuscript drafting and final approval.

Dr Grutters: contributed to the study concept and design, patient recruitment and data collection, data analysis and interpretation, manuscript preparation, and manuscript drafting and final approval.

Financial/nonfinancial disclosures: The authors have reported to CHEST that no potential conflicts of interest exist with any companies/organizations whose products or services may be discussed in this article.

Additional information: The e-Appendix can be found in the "Supplemental Materials" area of the online article.

\section{REFERENCES}

1. Paramothayan NS, Lasserson TJ, Jones PW. Corticosteroids for pulmonary sarcoidosis. Cochrane Database Syst Rev. 2005; (2):CD001114

2. Grutters JC, van den Bosch JM. Corticosteroid treatment in sarcoidosis. Eur Respir J. 2006;28(3):627-636.

3. Schutt AC, Bullington WM, Judson MA. Pharmacotherapy for pulmonary sarcoidosis: a Delphi consensus study. Respir Med. 2010;104(5):717-723.

4. Baughman RP, Nunes H. Therapy for sarcoidosis: evidencebased recommendations. Expert Rev Clin Immunol. 2012; 8(1):95-103.

5. Kiltz U, Braun J. Use of methotrexate in patients with sarcoidosis. Clin Exp Rheumatol. 2010;28(5)(suppl 61):S183-S185.

6. Lower EE, Baughman RP. The use of low dose methotrexate in refractory sarcoidosis. Am J Med Sci. 1990;299(3):153-157.

7. Webster GF, Razsi LK, Sanchez M, Shupack JL. Weekly lowdose methotrexate therapy for cutaneous sarcoidosis. J Am Acad Dermatol. 1991;24(3):451-454.

8. Vucinic VM. What is the future of methotrexate in sarcoidosis? A study and review. Curr Opin Pulm Med. 2002;8(5): 470-476.

9. Baughman RP, Winget DB, Lower EE. Methotrexate is steroid sparing in acute sarcoidosis: results of a double blind, randomized trial. Sarcoidosis Vasc Diffuse Lung Dis. 2000;17(1): 60-66.

10. Lower EE, Baughman RP. Prolonged use of methotrexate for sarcoidosis. Arch Intern Med. 1995;155(8):846-851.

11. Pacheco Y, Marechal C, Marechal F, Biot N, Perrin Fayolle $\mathrm{M}$. Azathioprine treatment of chronic pulmonary sarcoidosis. Sarcoidosis. 1985;2(2):107-113.

12. Müller-Quernheim J, Kienast K, Held M, Pfeifer S, Costabel U. Treatment of chronic sarcoidosis with an azathioprine/prednisolone regimen. Eur Respir J. 1999;14(5):1117-1122.

13. Lewis SJ, Ainslie GM, Bateman ED. Efficacy of azathioprine as second-line treatment in pulmonary sarcoidosis. Sarcoidosis Vasc Diffuse Lung Dis. 1999;16(1):87-92.

14. Baughman RP, Drent M, Kavuru M, et al; Sarcoidosis Investigators. Infliximab therapy in patients with chronic sarcoidosis and pulmonary involvement. Am J Respir Crit Care Med. 2006; 174(7):795-802

15. Hunninghake GW, Costabel U, Ando M, et al. ATS/ERS/WASOG statement on sarcoidosis. American Thoracic Society/European Respiratory Society/World Association of Sarcoidosis and Other Granulomatous Disorders. Sarcoidosis Vasc Diffuse Lung Dis. 1999;16(2):149-173.

16. Hedeker D, Gibbons RD. Longitudinal Data Analysis. Hoboken, NJ: John Wiley \& Sons; 2006.

17. Bernatsky S, Hudson M, Suissa S. Anti-rheumatic drug use and risk of serious infections in rheumatoid arthritis. Rheumatology (Oxford). 2007;46(7):1157-1160.

18. Arnold MH, O'Callaghan J, McCredie M, Beller EM, Kelly DE, Brooks PM. Comparative controlled trial of low-dose weekly 
methotrexate versus azathioprine in rheumatoid arthritis: 3-year prospective study. Br J Rheumatol. 1990;29(2):120-125.

19. Pagnoux C, Mahr A, Hamidou MA, et al; French Vasculitis Study Group. Azathioprine or methotrexate maintenance for ANCAassociated vasculitis. N Engl J Med. 2008;359(26):2790-2803.

20. Baughman RP, Lower EE. Alternatives to corticosteroids in the treatment of sarcoidosis. Sarcoidosis Vasc Diffuse Lung Dis. 1997;14(2):121-130.

21. Medicijnkosten website. http://www.medicijnkosten.nl. Accessed May 26, 2012.
22. Belgium Centre for Farmacotherapeutic Information website. http://www.bcfi.be. Accessed May 26, 2012.

23. Goossens HA, Ferech M, Vander Stichele R, Elseviers M; ESAC Project Group. Outpatient antibiotic use in Europe and association with resistance: a cross-national database study. Lancet. 2005;365(9459):579-587.

24. Dong XW, Zheng Q, Zhu MM, Tong JL, Ran ZH. Thiopurine S-methyltransferase polymorphisms and thiopurine toxicity in treatment of inflammatory bowel disease. World J Gastroenterol. 2010;16(25):3187-3195. 